\title{
Envelope Instabilities in Electron Cooling
}

\section{Abstract}

The envelope instabilities are considered as factors responsible for the observed limitations for a current of cooled particles.

\section{OBSERVATIONS}

Electron cooling is proved to be a way to increase a phase space density of heavy particles. However, its possibilities and limits are not compleatly studied. One of the unclear phenomena is the limit on the cooled beam intensity observed at several cooling facilities [1, 2, 3, 4]. The main features of the phenomenon experimentally studied in the IUCF cooler [1] are listed below.

1. Under the continuous stripping injection of protons in the cooler, the accumulated proton current was limited by a certain value independent on the injected current. The rate of the current increase was seen to be constant until just below the limiting current where the rate sharply dropped to zero. The phenomenon can be described as the beam lifetime being a highly nonlinear function of the beam intensity, see Fig. 1.

2. The operations with the bunched proton beam showed that the peak proton current, not the average one, is limited.

3. The beam current decreased smoothly between injection cycles, thus suggesting the beam scraping is not of concern.

4. The size of the cooled beam at the limit current was found to be very small, the rms radius $a_{c}=0.5 \mathrm{~mm}$, which is deeply inside the electron beam with the radius $a_{e}=1.2 \mathrm{~cm}$. The space charge tune shift in the cooled beam was found to be high and approximately constant during the accumulation, $\Delta Q \simeq 0.2$ [5].

5. The beam halo significantly increased near the accumulation threshold, see Fig. 2 [6].

6. Attempts to dilute the transverse emittance of the cooled beam by means of the applied transverse broad band noise resulted only in the decrease of the accumulated current [6].

7. Coherent transverse signals were not observed.

For the present moment, there is no satisfactory explanation of these facts. It is not clear, why instead of the gradual emittance growth proportionally to the peak current (to keep the tune shift constant), the life time of the protons sharply drops and the accumulation stops.

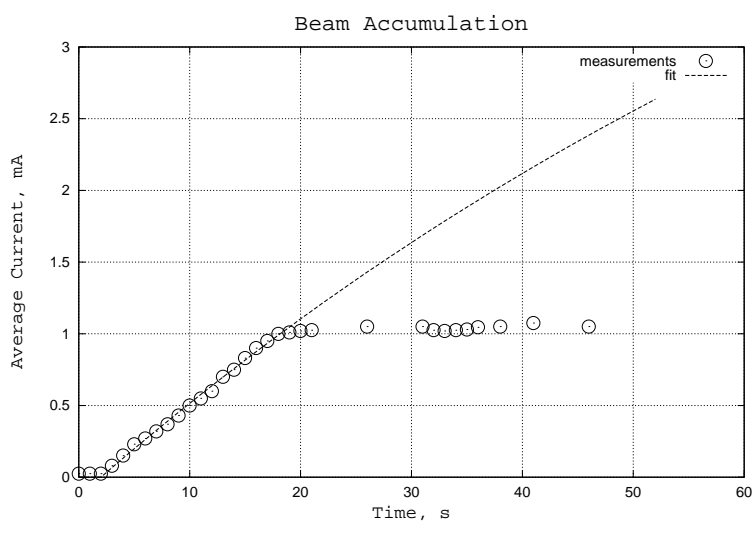

Figure 1: Accumulated current $I$ as a function of time $t$ during stripping injection with cooling accumulation (IUCF). Dots are the measurements, line is a fit $I \propto 1-\exp \left(-t / \tau_{0}\right)$ for the lifitime $\tau_{0}=100 \mathrm{~s}$.

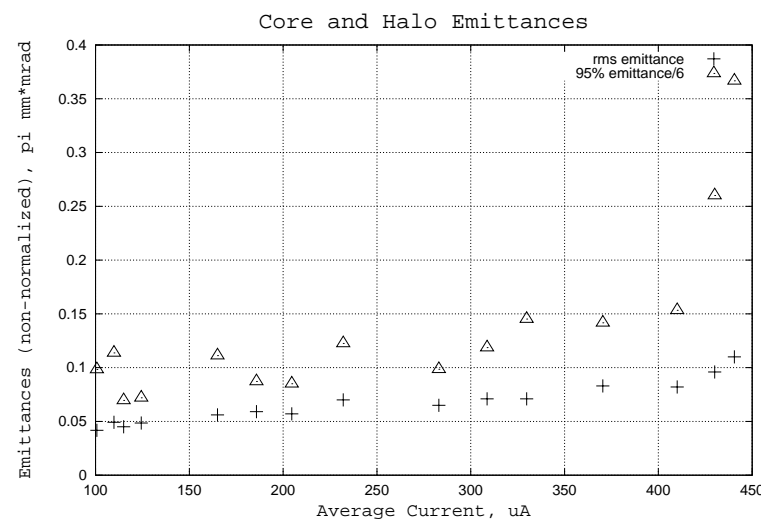

Figure 2: Beam core and halo measurements during the accumulation of bunched beam (IUCF). The upper set of points is $1 / 6$ of $95 \%$ emittance, and the lower set is rms emittance. If the transverse distribution were Gaussian, both sets would be equal. 
An attempt was made to explain these facts by a microwave electron-proton instability [4]. However, Ref [7, 8 ] found that the coherent electron-proton interaction introduces a stabilization in the proton beam motion under normal conditions.

The main hypothesis of this paper is that it is the envelope instability of the cooled beam which might be a reason for the described phenomenon.

\section{ENVELOPE INSTABILITIES}

Due to the periodic modulation of the focusing force, there is a possibility of parametric excitation of the beam secondorder (quadrupole) modes, resulting in the envelope instabilities [9, 10]. The instability take place when the space charge of the beam shifts frequency of one or both quadrupole modes to the parametrical resonance with the focusing structure. It means that the mode frequency referred to the structure frequency reaches half of an integer, $n / 2$, which approximately corresponds to the quarter of integer, $n / 4$, in terms of the single particle tune.

The structure period of a storage ring is, generally, the total circumference. Taking this into account, it can be concluded that a threshold of the envelope instability in the storage ring is reached when the space charge shift brings one of the betatron tunes to a quarter of an integer which sets the limit on the space charge tune shift $|\Delta \nu|<0.25$.

Strictly speaking, the envelope instabilities can be avoided for any space charge, if the storage ring is constructed from identical or almost identical cells. Such a storage ring has to obey the limit $\Delta \nu<N_{c} / 4$ which would be valid for any space charge if the number of cells $N_{c}$ is high enough, $N_{c}>4 \nu$. Due to the ring curvature, there is a minimal periodicity to satisfy this requirement. A storage ring of such a kind was suggested for experiments aimed to reach a crystalline state of a stored ion beam [11], the minimal periodicity was found to be $N_{c}=8$.

Apart from this special case, the restriction on the envelope instability coinsides with the conventionally accepted single-particle limit on the space charge tune shift, $|\Delta \nu|<$ 0.25 [12].

The numerous simulations show that if the beam is unstable initially, the coherent oscillations start to grow so that after a few structure periods the beam emittance is diluted enough to be in the stable area.

Situation would be different, were the beam under the cooling. Cooling would not allow the beam just permanently stay in the stable area: it would continue to shrink the emittance and lead the beam again to the instability threshold.

\section{COLD BEAM AS A SOURCE OF NOISE}

Thus, the electron cooling and the envelope instability can be considered to be in a dynamic equilibrium resulted in keeping the cooled beam at the threshold of the instability. The electric field of the oscillating beam core acts on the outer, hot protons. The perturbation of the proton transverse energy is mainly contributed by such betatron phases when it passes the oscillating beam close enough, at impact parameters less or about the beam radius $a_{c}$. For a proton with an amplitude $a \gg a_{c}$, this happens in average once per time interval

$$
\Delta t \simeq a / a_{c} \omega_{b}^{-1} \gg \omega_{b}^{-1},
$$

where $\omega_{b}=\nu \omega_{0}$ is the betatron frequency, $\omega_{0}$ is the revolution frequency. Every time when the proton passes near the oscillating core, its angles are changed (increased or decreased) by about

$$
\Delta \theta \simeq \frac{2 \rho_{p} r_{p} c}{\beta \gamma^{3} a_{c}} \frac{\tilde{a}_{c}}{a_{c}} \omega_{b} \tilde{\tau}^{2},
$$

where $\rho_{p}$ is the proton beam linear density, $r_{p}$ is the proton classical radius, $c$ is the spead of light, $\beta$ and $\gamma$ are the relativistic factors,

$$
\tilde{\tau} \simeq a_{c} / a \omega_{b}^{-1}
$$

is a time of the interaction, $\tilde{a}_{c} / a_{c} \simeq 1$ is the relative amplitude of the beam size oscillations responsible for the considered interaction. The quadratic dependence on the interaction time, $\Delta \theta \propto \omega_{b} \tilde{\tau}^{2}$, is related to the fact that the change of the proton transverse energy is caused by the free oscillations of the beam core, which contributes $\propto \omega_{b} \tilde{\tau}$. The constant field changes the momentum components, but not the total energy.

The main hypothesis of the presented model is that this energy change of the hot proton can be considered as random. In other words, it is assumed that the oscillating fields of the beam seen by the proton when it passes the core, are compleatly unrelated to the fields seen on the previous pass. This assumption looks acceptable due to the following factors:

- A correlation time for oscillations of a system near its instability threshold is typically not more than several periods of the oscillations.

- The longitudinal correlations of the envelope oscillations extend not longer than for the core radius. Due to the longitudinal velocity of the proton, every pass it sees the core at various longitudinal coordinates where the fields are not correlated.

- Finally, this assumption of random interactions can be modified: it could be assumed that the correlation time is by a certain factor $f_{r}$ higher than the average time between consequent interactions. However, the calculations show that the resulted dependence on this factor is very weak, it enters as $\propto f_{r}^{2 / 7}$.

Thus, the interactions of the remote proton with the oscillating beam core are considered as independent inelastic scatterings causing a diffusion of the proton energy. The proton angle changes due to the simultaneous action of this diffusion with the coefficient

$$
D=(\Delta \theta)^{2} / \Delta t=\frac{4 \rho_{p}^{2} r_{p}^{2} a_{c}^{3} c^{2}}{\omega_{b} a^{5} \beta^{2} \gamma^{6}}
$$


and the electron cooling with the rate

$$
\lambda=\frac{4 \pi n_{e} r_{e} r_{p} L_{C} \eta c}{\theta^{3} \beta^{3} \gamma^{5}}
$$

where $n_{e}$ is the electron beam density, $r_{e}, r_{p}$ are the electron and proton classical radii, $L_{C}$ is the Coulomb logarithm and $\eta$ is the cooling length related to the ring circumference, $\theta=$ $a \omega_{b} /(\beta c)$ is a proton angle amplitude.

Evolution of the proton distribution $f(\theta)$ under an influence of the cooling and diffusion is described by the FokkerPlank equation:

$$
\frac{\partial f}{\partial t}=\frac{\partial}{\partial \theta}\left(-\lambda \theta f+\frac{D}{2} \frac{\partial f}{\partial \theta}\right) .
$$

This determines an equilibrium distribution:

$$
f(\theta)=f_{0} \exp \left(-2 \int_{0}^{\theta} \frac{\lambda\left(\theta^{\prime}\right) \theta^{\prime} d \theta^{\prime}}{D\left(\theta^{\prime}\right)}\right)
$$

with $f_{0} \simeq 1 / \theta_{c}$, where $\theta_{c}=a_{c} \omega_{b} /(\beta c)$ is the angle amplitude of the core particles. Assuming the aperture limit $\theta=\theta_{a}=a_{m} \omega_{b} /(\beta c)$, the life time $\tau$ follows from (4), (5):

$$
\tau^{-1} \simeq \lambda_{a} \theta_{a} \exp \left(-2 \int_{0}^{\theta_{a}} \frac{\lambda(\theta) \theta d \theta}{D(\theta)}\right), \lambda_{a}=\lambda\left(\theta_{a}\right) .
$$

The lifetime (6) contains current-dependent terms in the exponent, so it is highly nonlinear function of the beam intensity.

Assuming a finite storage time $\tau_{s}=d \ln N / d t$, and taking into account the dependencies (2), (3) the intensity limitation follows:

$$
\frac{\lambda_{a} \theta_{a}^{2}}{2 D_{a}}=L_{a}, \quad L_{a}=\ln \left(\lambda_{a} \tau_{s} a_{m} / a_{c}\right), D_{a}=D\left(\theta_{a}\right) .
$$

Then, the proton beam linear density $\rho_{p}$ and its radius $a_{c}$ determine the space charge tune shift

$$
\Delta \nu=\frac{\rho_{p} r_{p} R^{2}}{2 \nu \beta^{2} \gamma^{3} a_{c}^{2}}
$$

where $R$ is the storage ring average radius. The threshold condition (7) can be resolved for the proton beam peak current $I_{p}=\rho_{p} e \beta c$ and presented in the following form:

$$
I_{p}=e c \beta \gamma^{11 / 7} a_{m}^{8 / 7}\left(\frac{\pi n_{e} \eta L_{C}}{2 L_{a}} \frac{r_{e}}{r_{p}}\right)^{2 / 7}\left(\frac{2 \nu \Delta \nu \beta^{2}}{r_{p} R^{2}}\right)^{3 / 7} .
$$

The obtained threshold (9) can be calculated for the parameters of the IUCF cooler. The aperture $\log$ arithm $L_{a}$ (7) calculated with $a_{m}=1.5 \mathrm{~cm}, a_{c}=0.5 \mathrm{~mm}, \lambda_{a}=0.3 \mathrm{~s}^{-1}$, $\tau_{s}=18 \mathrm{~s}$, comes out $L_{a}=6$. Substituting $I_{e}=\rho_{e} e \beta c=$ $400 \mathrm{~mA}, a_{e}=1.2 \mathrm{~cm}, \beta=0.3, L_{C}=3, \eta=0.03, \nu=$ $4, \Delta \nu=0.25, R=15 \mathrm{~m}$, it gives $I_{p}=6 \mathrm{~mA}$, in an agreement with the observations [1]. Assuming the same aperture limitations and electron current, approximately same numbers $( \pm 50 \%)$ comes out for the proton threshold current in CELSIUS and $\mathrm{Kr}^{34+}$ current in SIS.

So we may conclude about an agreement between this model and the observations.

\section{HOW TO INCREASE THIS THRESHOLD}

A slight misalignment $\psi$ introduced in both transverse directions between the electron and proton beam could increase the threshold. In this case the protons with smaller angles cannot be accumulated, this phase space area is unstable for the single particle motion (the so-called monochromatic instability). The angle provided in both transverse planes should be higher than the threshold beam angles $\theta_{c}$ but smaller than the angle at the injection $\theta_{i}$ :

$$
\theta_{c}<\psi_{x, y}<\theta_{i}
$$

The higher is the misalignment, the higher is both the threshold current and the temperature of the accumulated protons.

An improvement with the similar restrictions (10) could be reached by means of the electron cooler with a hollow cathode $[1,13]$.

\section{REFERENCES}

[1] T. Ellison et al., "Cooled Beam Intensity Limits in the IUCF Cooler", in Proc. of Workshop on Beam Cooling and Related Topics, Montreux, Oct. 1993, CERN 94-03, p. 377.

[2] D. Reistad et al, "Measurements of Electron Cooling and 'Electron Heating' at CELSIUS', ibid, p. 183.

[3] M. Steck et al., "Commisioning of the Electron Cooling Device in SIS", in Proc. EPAC'98, Stokholm, p. 550.

[4] V.Parkhomchuk, "Limitation of Ion Beam Intensity in Electron Cooling Systems", in Proc. HEACC'98, Dubna, Russia.

[5] S. Nagaitsev et al., NIM A, 391, p.32 (1997).

[6] S. Nagaitsev, PhD Thesis, Indiana University, 1995.

[7] A. Burov, Part. Accel., 57, 131 (1997).

[8] D. Pestrikov, NIM A, 412, p.283 (1998).

[9] I Hofmann, L. J. Laslett, L. Smith and I. Haber, Part. Accel., 13, 145 (1983)

[10] J. Struckmeier and M. Reiser, Part. Accel., 14, 227 (1984)

[11] L. Tecchio et al. "CRYSTAL Ring, Feasibility Study”, LNLINFN Rep., Legnaro, Italy (1996).

[12] M. Reiser, Theory and Design of Charged Particle Beams, J. Wiley \& Sons Inc., 1994.

[13] A. Sharapa, A. Shemyakin, NIM A 336 p.6 (1993). 